\title{
Moderators of relative confidence calibration: A meta-analysis of the across-subject relationship between confidence and accuracy
}

\author{
Sunny Jin, Paul Verhaeghen \& Dobromir Rahnev \\ School of Psychology, Georgia Institute of Technology, Atlanta, GA
}

Keywords: confidence, metacognition, perceptual decision making, memory, calibration, bias

\section{Acknowledgements}

This work was supported by the National Institute of Health (award:

R01MH119189) and the Office of Naval Research (award: N00014-20-1-2622).

\section{Correspondence}

Dobromir Rahnev

Georgia Institute of Technology

654 Cherry Str. NW

Atlanta, GA 30332

E-mail: rahnev@psych.gatech.edu 


\begin{abstract}
If one friend confidently tells us to buy product A while another friend thinks that product B is better but is not confident, we may go with the advice of our confident friend. Should we? The relationship between people's confidence and accuracy has been of great interest in many fields, especially in the context of high-stakes situations like eye-witness testimony, but there is still little consensus about how much we should trust someone's overall level of confidence. Here we examine the across-subject relationship between average accuracy and average confidence in 214 unique datasets from the Confidence Database. This approach allows us to empirically address this issue with unprecedented statistical power and check for the presence of various moderators. We find that the across-subject correlation between average accuracy and average confidence in this sample is $\mathrm{R}=.22$. Importantly, this relationship is much stronger for memory than for perception tasks, as well as for confidence scales with fewer points. These results show that we should take one's confidence seriously (and perhaps buy product A) and suggest several factors that moderate the relative consistency of how people make confidence judgments.
\end{abstract}




\section{Introduction}

Expressing an appropriate level of confidence is of utmost importance in many facets of human life. From the perspective of the individual, one's own confidence helps determine whether to commit to a decision or either gather more information (Desender et al., 2018) or seek advice from others (Pescetelli \& Yeung, 2021). Our own confidence also helps us determine whether we have sufficient expertise in a particular domain or more learning is required (Dautriche et al., 2021).

Yet, confidence perhaps becomes even more important in the interpersonal domain. For example, the level of confidence in a diagnosis expressed by medical personnel can have a substantial effect on how the diagnosis is received and its ultimate impact on patients (Yang \& Thompson, 2010). Similarly, the confidence expressed in eyewitness testimonies can have a large impact on the outcome of a trial (Tenney et al., 2008). Perhaps due to the enormous stakes of such testimonies, there is a lively debate on how trustworthy confidence ratings of eye witnesses really are (Berkowitz et al., 2020; Juslin et al., 1996; Loftus \& Greenspan, 2017; Penrod \& Cutler, 1995; Wixted \& Wells, 2017). As these examples demonstrate, understanding how much we can trust the confidence expressed by others has important implications for many domains as disparate as law, medicine, and education.

The trustworthiness of confidence has been extensively studied by eliciting a single (or at most a few) decisions from each participant. While this approach mimics closely many real-world situations (e.g., eyewitness testimony), it sheds virtually no 
light on the question of how well people's overall confidence reflects their overall accuracy on a task where many decisions may be involved. This is because a single decision is either correct or incorrect, but, in reality, people vary on their underlying performance ability in a continuous manner (e.g., 70 vs. $80 \%$ correct on a 2 -choice task). Therefore, the question of how much we should trust confident vs. nonconfident people should also be addressed by examining situations where each participant completes many trials that involve both a primary decision and a confidence judgment so that their overall accuracy and confidence can be assessed.

Here we adopted this approach with unprecedented statistical power by taking advantage of the recently published Confidence Database (Rahnev et al., 2020). This database includes many datasets featuring confidence ratings that come primarily from traditional laboratory studies where each participant completes hundreds of trials. Importantly, the datasets vary in many aspects including the domain of study (e.g., perception or memory), the confidence scale used, the presence of trial-by-trial feedback, etc. This variability allowed us to further examine the moderators that makes overall confidence more or less predictive of overall accuracy. To anticipate, we found that average confidence and average accuracy correlate at $\mathrm{R}=.22$ with higher correlations being observed in memory studies and with confidence scales that have fewer points. 


\section{Methods}

\section{Data selection}

The data for analysis was taken from the Confidence Database (Rahnev et al., 2020), a large collection of datasets that include confidence ratings (available at https://osf.io/s46pr/). The data in the Confidence Database came from dozens of labs across 16 countries on five continents. Correspondingly, the individual datasets vary in many ways such as the domain of study, the specific task, the number of participants, the number of trials per participant, how confidence was collected, etc. Most perception tasks involve a discrimination between two stimulus categories such as left- vs. right-tilted gratings or dot motion that moves to the left vs. right. Most memory tasks involve the standard procedure of studying a list of items (e.g., word, pictures, etc.) and providing old/new judgments with confidence during a later recognition test. However, while these common designs account for the majority of the datasets, many specific datasets feature unique designs that depart from the common tasks described above.

The complete database was downloaded in October 2019 (note that several more datasets have been added to it after that date) and featured 145 separate datasets. One dataset ("Dildine_unpub") used a task where objective performance could not be computed and was therefore excluded. Another dataset ("Zylberberg_2016") had only three participants and was therefore also excluded (for the purposes of conducting a meta-analysis, we required that each dataset has at least four 
participants; see below). All analyses were thus performed on the remaining 143 original datasets.

Several datasets included the data from the initial training prior to the main experiment but most datasets did not include these data. Therefore, for consistency, we excluded the training data from all datasets. Due to the vast differences between the tasks used in different datasets, it was impossible to devise a standardized exclusion criteria for individual participants. Therefore, all participants from each dataset were included in the analyses.

\section{Data preprocessing}

While most datasets featured a single task completed by all participants, this was not universally true. In fact, several datasets featured different tasks (or conditions), with each task completed by a separate subset of participants. However, task differences between participants could potentially bias the relationship between average accuracy and average confidence across the whole group. Therefore, to avoid such biases, we manually examined each dataset and split each original dataset that features multiple tasks (or conditions) that were completed by separate subsets of participants into separate datasets (subsequently we use the term "original dataset" to refer to the original datasets found in the Confidence Database, and the term "dataset" to refer to the datasets obtained after the splitting process). This process identified 34 original datasets with multiple tasks (19 with two tasks each, seven with three tasks each, three with four tasks each, three with six tasks 
each, one with eight tasks, and one with 10 tasks). Details on these original datasets and how they were split are provided in the Supplementary Methods. This process resulted in a total of 216 datasets. However, two of these new datasets only included three participants and were therefore excluded, thus leaving us with a total of 214 individual datasets for all remaining analyses.

\section{Analyses}

For each of the 214 final datasets, we computed the average accuracy and average confidence for each participant. Two hundred out of the 214 datasets featured discrimination tasks where participants chose one response among several (typically two) options. For such cases, accuracy was computed based on whether a response was the same as the correct answer. The remaining 14 datasets, however, featured estimation tasks where, for example, participants had to give a numerical guess regarding the duration or orientation of a continuous stimulus. In such cases, accuracy was computed as the deviation between the response and the correct answer. However, since small deviations correspond to better performance, for these datasets, "accuracy" was defined as the deviation between the response and the correct answer multiplied by -1 , thus ensuring that higher accuracy corresponds to higher performance.

Once the average accuracy and average confidence were computed for each participant in a dataset, we performed a Pearson correlation between these two quantities. We then z-transformed the resulting R-values before conducting further 
statistical analyses (though, for display purposes, we plot the original R-values in all figures).

The primary analysis consisted of a meta-analysis across all 214 z-transformed Rvalues. The meta-analysis weighted each $\mathrm{z}$-value based on its variance, $\mathrm{z}_{\mathrm{var}}$, that is equal to $1 /(n-3)$, where $n$ is the number of participants. This value is only defined for $n \geq 4$, which necessitated the exclusion of all datasets with $n \leq 3$.

Additional analyses investigated whether different experimental factors moderated the relationship between average confidence and average accuracy. Based on the information provided as part of the Confidence Database, we were able to identify six factors for which we could find relevant information for every dataset. These factors were: (1) the confidence scale used (confidence scales had 2, 3, 4, 5, 6, 9, 11 points or were continuous), (2) domain of study (whether the study used a memory task, a perception task, or a task, "other," that was not from either domain such as cognitive or motor tasks), (3) timing of the confidence judgments (simultaneous with the decision or after the decision), (4) the presence of trial-by-trial feedback (present or absent), (5) the type of task (discrimination or estimation), and (6) the average number of trials per participant. We performed a mixed-effects metaanalysis with all six factors, using the metafor package in R (Viechtbauer, 2010), and examined whether any factor was a significant moderator of the correlation between average accuracy and average confidence. 
We assessed the inter-study variation in effect sizes via the Q (Hedges \& Olkin, 1985) and $I^{2}$ (Cooper, 2017) statistics. We further checked for publication bias by performing a regression test for funnel plot asymmetry.

\section{$\underline{\text { Data and code }}$}

All data and codes for preprocessing and analysis are available at https://osf.io/kpe75/. 


\section{Results}

We investigated the across-subject correlation between average confidence and average accuracy using 214 separate datasets extracted from the Confidence Database (Rahnev et al., 2020). Details regarding the datasets are available in Figure 1. There were 9,191 total participants and 3,899,139 total trials used for estimating the correlations.
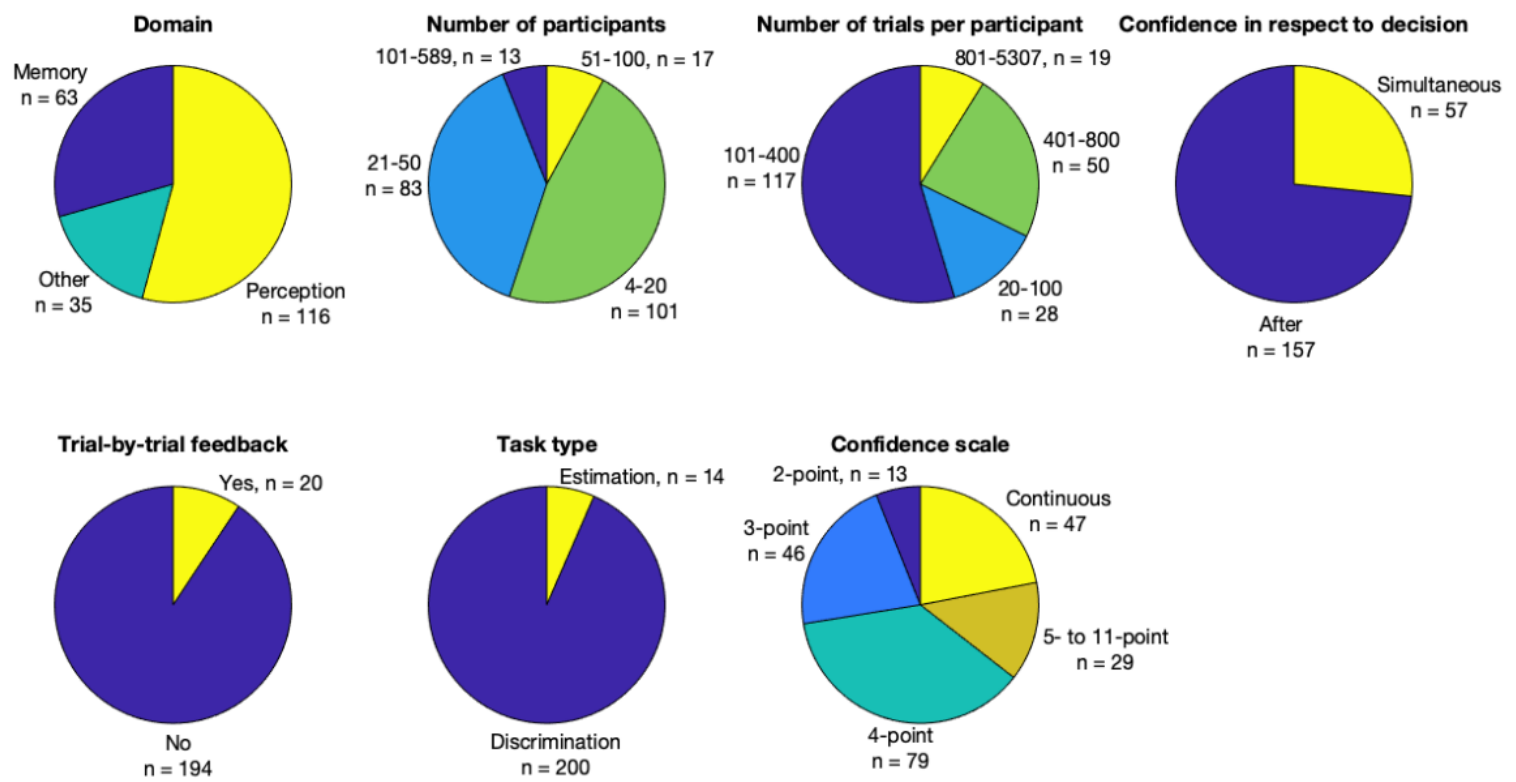

Figure 1. Dataset information. Pie charts with information regarding the different datasets. The "Other" domain includes cognitive and motor tasks. The great majority of datasets were relatively small with typical size of less than 50 participants. On the other hand, each participant performed many trials (average $=506$ trials per participant). Most datasets collected confidence after the decision, did not provide trial-by-trial feedback, and employed discrimination tasks (where participants chose an answer among, typically, two alternatives). There was a lot of variability in the confidence scale used with 3-point, 4-point, and continuous scales being the most frequent.

We first computed the meta-analytic average correlation across all 214 datasets. We found a highly significant correlation $(\mathrm{R}=.22, \mathrm{p}<.0001,95 \% \mathrm{CI}=[.18, .26]$; Figure 
2). Further, we found high heterogeneity among the datasets $\left(I^{2}=67.67 \%\right)$ that was significantly above chance $(Q(213)=649.99, \mathrm{p}<0.001)$, suggesting that one or several moderators may further determine the strength of the correlation between average accuracy and average confidence. Repeating the analyses by ignoring sample size (via a simple $\mathrm{t}$-test) produced very similar results $(\mathrm{R}=.20, \mathrm{t}(213)=$ 9.26, $\mathrm{p}=2.3^{*} 10^{-17}$, Cohen's $d=.63$ ). An additional meta-analysis performed only on the 113 datasets with a sample size $\mathrm{n}>20$ also produced very similar results $(\mathrm{R}=$ $.20, \mathrm{p}<.0001,95 \% \mathrm{CI}=[.15, .25])$.

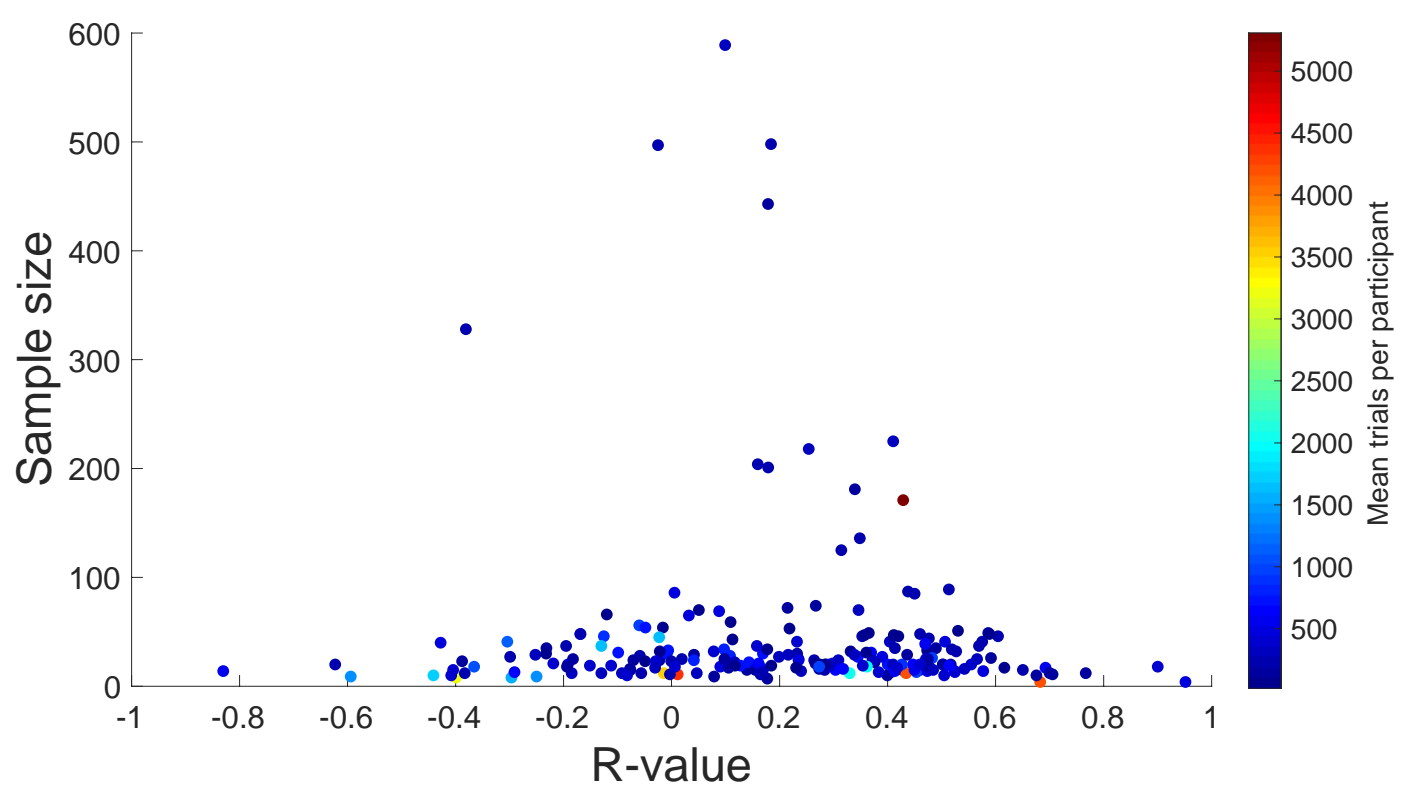

Figure 2. Correlation coefficient (R-value) as a function of sample size and mean trials per participant. The strength of the correlation between average accuracy and average confidence is plotted against sample size. Colors indicate the mean trials per participant for each dataset. Each circle is a separate dataset.

To our knowledge, none of the data in the Confidence Database were collected with the purpose of identifying the correlation between average confidence and average 
accuracy. Therefore, we expected little to no publication bias for the datasets in the current meta-analysis. Indeed, Figure 2 reveals no clear asymmetry, which would be indicative of publication bias. The lack of asymmetry was further confirmed by a mixed-effects meta-regression test for funnel plot asymmetry $(\mathrm{z}=.51, p=.61)$.

Having established the existence of a significant correlation between average accuracy and average confidence, we examined whether this relationship depends on any moderators. To this end, we performed a mixed-effects meta-analysis with factors confidence scale use (discrete vs. continuous), domain of study (perception vs. memory vs. other), timing of the confidence judgments (with vs. after the decision), presence of trial-by-trial feedback (present vs. absent), type of task (discrimination vs. estimation), and average number of trials per participant.

We found a significant effect of the confidence scale with coarser scales (i.e., scales with fewer points) resulting in higher R-values (Figure 3A and Table 1). Specifically, continuous scales produced significantly lower R-values compared to discrete scales $(\mathrm{z}=2.59, p=.0096)$. A similar result was obtained in an alternative mixed-effects meta-analysis where 2-, 3-, and 4-points confidence scales were compared to scales with at least 5 points $(\mathrm{z}=2.84, p=.0045)$. We further performed a non-parametric Spearman correlation between the number of points on the confidence scale and Rvalues and found it to be significantly negative (rho $=-.205, p=.0025)$. These results suggest that coarser confidence scales give rise to higher correlations between average accuracy and average confidence. 

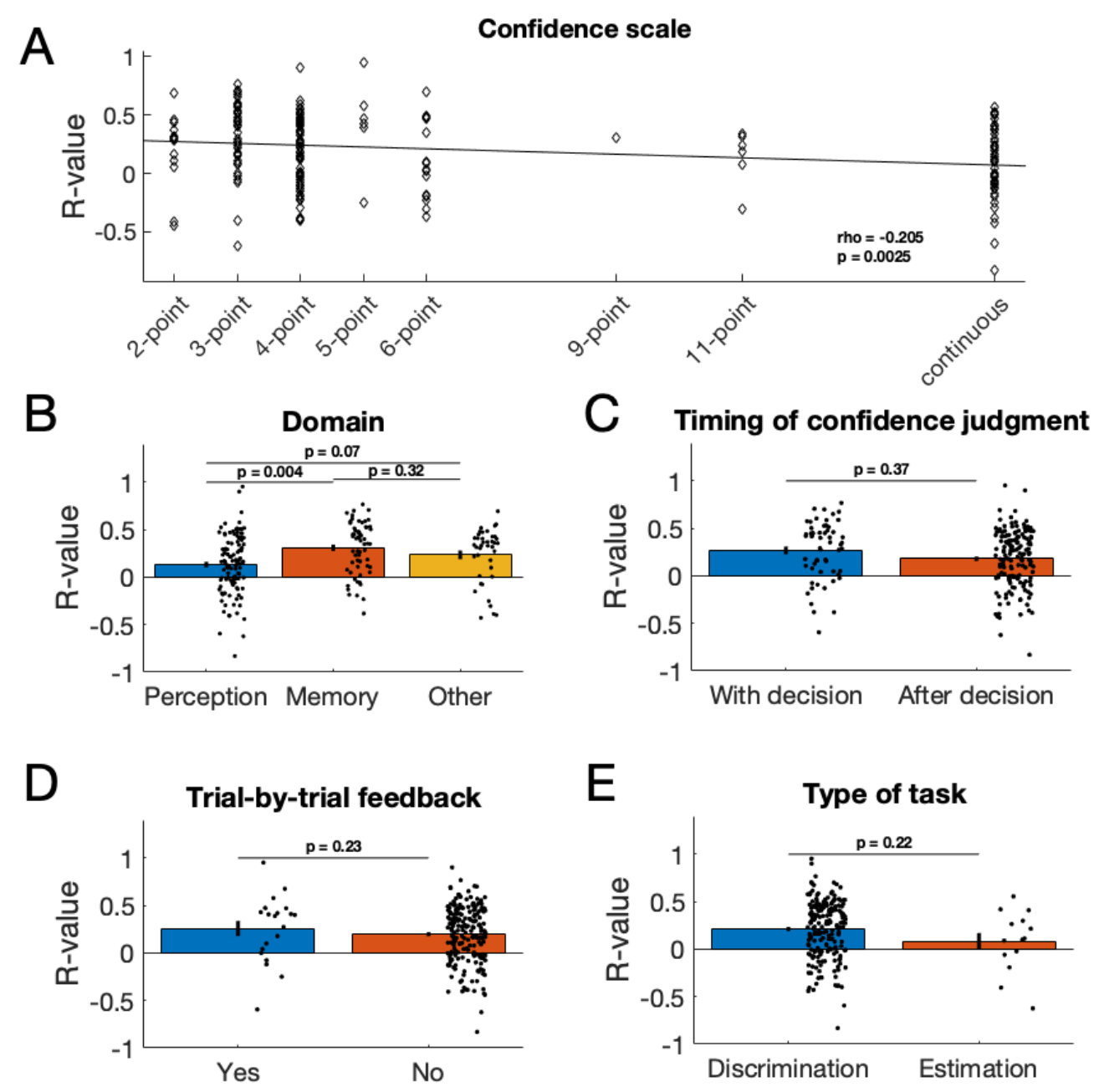

Figure 3. Moderators of the relationship between average accuracy and average confidence. The strength of the correlation (R-value) between average accuracy and average confidence was (A) higher for coarser confidence scales (that is, scales with fewer points), (B) highest for memory tasks and lowest for perception tasks, and not significantly different for datasets that differed on (C) the timing of the confidence judgment, (D) the presence of trial-by-trial feedback, and (E) the type of task. Error bars show S.E.M. and each diamond/circle represents one dataset. The $\mathrm{p}$-values are derived from a (non-meta-analytic) Spearman correlation in (A) and from mixed-effects meta-analyses in (B-E).

\begin{tabular}{lrrrr}
\hline & $k$ & $R$ & $\begin{array}{r}\text { Lower limit } \\
\text { of 95\% CI }\end{array}$ & $\begin{array}{r}\text { Upper limit } \\
\text { of 95\% CI }\end{array}$ \\
\hline $\begin{array}{l}\text { Domain } \\
\text { Memory } \mathrm{a}^{*}\end{array}$ & 214 & .22 & .18 & .26 \\
\hline
\end{tabular}




\begin{tabular}{|c|c|c|c|c|}
\hline Perception ${ }^{\mathrm{b}^{*}}$ & 111 & .13 & .07 & .18 \\
\hline Other ${ }^{a^{*}}$ & 40 & .24 & .16 & .32 \\
\hline \multicolumn{5}{|c|}{ Trial-by-trial feedback } \\
\hline Yes $^{a *}$ & 20 & .26 & .09 & .42 \\
\hline $\mathrm{No}^{\mathrm{a} *}$ & 194 & .22 & .17 & .26 \\
\hline \multicolumn{5}{|c|}{ Timing of confidence judgment } \\
\hline With decisiona* & 57 & .31 & .22 & .40 \\
\hline After decision ${ }^{\mathrm{b} *}$ & 157 & .19 & .09 & .28 \\
\hline \multicolumn{5}{|l|}{ Confidence scale } \\
\hline Discrete $^{\mathrm{a} *}$ & 167 & .26 & .22 & .30 \\
\hline Continuous ${ }^{\mathrm{b}}$ & 47 & .08 & -.03 & 19 \\
\hline \multicolumn{5}{|l|}{ Type of task } \\
\hline Discriminationa* & 200 & .23 & .19 & .27 \\
\hline Estimation $^{\mathrm{a}}$ & 14 & .10 & -.10 & .29 \\
\hline
\end{tabular}

Table 1. Moderators of the relationship between average accuracy and average confidence. Asterisks denote significant effects at $p<.05$. Average effect sizes with different superscripts are significantly different from each other (except for the comparison between Perception and Other, which is only marginally significant). $k$, number of datasets; CI, Confidence Interval.

In addition, we found that the domain of study is also a strong moderator of the strength of the correlation between average accuracy and average confidence (Figure 3B). Specifically, perception studies produced the lowest R-value $(R=.13)$, which was significantly lower than in memory studies $(\mathrm{R}=.35, \mathrm{z}=2.92, p=.0035)$ and marginally lower than in studies from other domains $(\mathrm{R}=.24, \mathrm{z}=1.81, p=.07)$. Studies from memory and other domains were not significantly different from each other $(\mathrm{z}=-1.01, p=.32)$.

No other moderator was found to be significant including the timing of the confidence judgment (with or after the primary decision, $\mathrm{z}=.90, p=.37$; Figure $3 \mathrm{C}$ ), the existence of trial-by-trial feedback $(\mathrm{z}=1.21, p=.23$; Figure 3D), the type of task (discrimination or estimation, $\mathrm{z}=1.23, p=.22$; Figure $3 \mathrm{E}$ ), or the mean number of trials per participant in a dataset $(\mathrm{z}=.65, p=.52)$. 


\section{Discussion}

We investigated the question of how much one should trust the judgment of a confident person compared to that of someone who is not confident. To address this question, we performed a meta-analysis on 214 datasets derived from the Confidence Database. For each dataset, we computed the average accuracy and average confidence for each participant, and then correlated these values across subjects. We found that this correlation was significantly positive and was of moderate size $(\mathrm{R}=.22)$. Further, the strength of the relationship was moderated by both the domain of the study and the coarseness of the confidence scale used. These results begin to reveal not just the overall strength of the across-subject relationship between accuracy and confidence, but also the moderators of this relationship.

It is important to appreciate exactly what drives the correlation between average accuracy and average confidence. This correlation reflects the relative calibration of confidence ratings across participants. In other words, it shows whether people's confidence ratings are well calibrated relative to each other. For example, it is possible to achieve high relative calibration even if there's a strong over- or underconfidence bias in the whole group as long as this bias is consistent across participants (Fleming \& Lau, 2014). Conversely, within-subject confidence ratings may be maximally informative (i.e., they could show no metacognitive inefficiency; Shekhar \& Rahnev, 2021a, 2021b), but different over- or under-confidence bias for different people could still result in low across-subject correlations. Therefore, the R-values examined here should not be interpreted as showing "how informative 
confidence is" in a general sense, but rather how well people's confidence ratings are calibrated relative to other people in the group. Returning to our example from the Abstract, if two friends give us conflicting advice, going with the advice of the more confident friend relies on the assumption that the confidence ratings expressed by these two friends are well calibrated relative to each other. Our findings indeed confirm that, in the absence of additional information, following the advice of the more confident friend is indeed the best strategy.

A related question is how to judge the magnitude of the meta-analytic correlation of $\mathrm{R}=.22$. Traditionally, this correlation would be considered to be somewhere between small $(\mathrm{R}=.1)$ and medium $(\mathrm{R}=.3)$ (Cohen, 1988). However, this characterization has been criticized as nonsensical and has been reportedly disavowed by Cohen himself (Funder \& Ozer, 2019). Instead, Funder and Ozer proposed a classification according to which $\mathrm{R}=.2$ "indicates a medium effect that is of some explanatory and practical use even in the short run", $\mathrm{R}=.3$ "indicates a large effect that is potentially powerful in both the short and the long run," and R > .4 indicates a "very large effect size" that, in the context of psychological research, is likely to be an overestimation. They argue that this classification matches intuitively-understood correlations such as the effectiveness of antihistamines on sneezing $(\mathrm{R}=.14)$, the higher weight of men compared to women $(\mathrm{R}=.26)$, and the lower average annual temperatures at higher elevations $(\mathrm{R}=.34)$. In this context, the observation of $\mathrm{R}=.22$ should be thought of as a medium effect, while the correlation of $\mathrm{R}=.35$ for memory studies should be seen somewhere between a 
large and a very large effect size. In other words, our results should be interpreted as showing overall good relative calibration of confidence that becomes especially strong in the context of memory tasks.

To our knowledge, the current paper represents the first meta-analysis of the relationship between average accuracy on a task and average confidence obtained on a trial-by-trial basis over the course of the same task. Nevertheless, our results are in line with the large literature on the relationship between objective and subjective ability estimates. For example, a meta-synthesis of 22 published metaanalyses of the relationship between self-evaluations and objective performance measures found an average correlation of $\mathrm{R}=.29$, with 18 of the meta-analyses reporting a correlation between .19 and .39 (Zell \& Krizan, 2014). Similarly, there has been a lot of work on the reliability of confidence judgments in eyewitness testimony with one meta-analysis (Sporer et al., 1995) finding an average correlation between the accuracy and confidence of identification of $\mathrm{R}=.29$ (though this correlation rose to $\mathrm{R}=.41$ when only positive identifications were considered). Therefore, it appears that the correlation between accuracy on a laboratory task and average confidence obtained on a trial-by-trial basis in the same task is largely consistent with the relationship between objective and subjective performance across a variety of fields.

One of the critical findings of the current meta-analysis concerns the moderators of the relationship between average accuracy and average confidence. We found that 
this relationship is roughly three times stronger for memory studies $(\mathrm{R}=.35)$ compared to perception studies $(\mathrm{R}=.13)$. We suspect that this difference may reflect participants' familiarity with the two types of tasks. Indeed, most people have plenty of experience with communicating their certainty in faint and unreliable memories, but few have sufficient experience with communicating their confidence for perceptual stimuli near the psychophysical threshold. As such, it is natural for people's confidence to be well calibrated relative to other people's confidence for memory but not perception tasks. In a similar fashion, it is likely that coarse confidence scales (that is, scales with few options) are likely to be interpreted by different people in a manner that is relatively more consistent across participants than detailed confidence scales (that is, scales with many options or continuous responses). Nevertheless, both of these explanations remain speculative and therefore require direct empirical validation.

We failed to find other significant moderators of the relationship between average accuracy and average confidence. Nevertheless, it should be noted that we had relatively little power for three of the moderators: the presence of trial-by-trial feedback, the type of task, and the timing of the confidence judgment. There were numerical differences in the R-values in all three cases with higher correlation coefficients observed in the presence of trial-by-trial feedback, discrimination (as opposed to estimation) tasks, and confidence ratings given simultaneously with (as opposed to after) the decision. The numerical difference in the context of trial-bytrial feedback is consistent with the findings of a recent large study that found a 
significant increase in the accuracy-confidence correlation in the presence of trialby-trial feedback (Haddara \& Rahnev, 2021). It is therefore possible that some of the factors that were not significant in the current meta-analysis may nonetheless be important for the size of the correlation between average accuracy and average confidence.

In conclusion, we performed a large meta-analysis on the relationship between average accuracy and average confidence in standard laboratory tasks and found a moderately strong relationship $(\mathrm{R}=.22)$ that is moderated by the domain of the study and the coarseness of the confidence scale. These findings provide important clues regarding how people interpret confidence ratings, and how consistent these interpretations are across participants. 


\section{References}

Berkowitz, S. R., Garrett, B. L., Fenn, K. M., \& Loftus, E. F. (2020). Convicting with confidence? Why we should not over-rely on eyewitness confidence. Memory. https://doi.org/10.1080/09658211.2020.1849308

Cohen, J. (1988). Statistical power analysis for the behavioral sciences (2nd ed.). Erlbaum.

Cooper, H. (2017). Research synthesis and meta-analysis: a step-by-step approach (5th editio). Sage.

Dautriche, I., Rabagliati, H., \& Smith, K. (2021). Subjective confidence influences word learning in a cross-situational statistical learning task. Journal of Memory and Language, 1-38.

Desender, K., Boldt, A., \& Yeung, N. (2018). Subjective Confidence Predicts Information Seeking in Decision Making. Psychological Science, 29(5), 761-778. https://doi.org/10.1177/0956797617744771

Fleming, S. M., \& Lau, H. C. (2014). How to measure metacognition. Frontiers in Human Neuroscience, 8. https://doi.org/10.3389/fnhum.2014.00443

Haddara, N., \& Rahnev, D. (2021). The impact of feedback on perceptual decision making and metacognition: Reduction in bias but no change in sensitivity. Psychological Science.

Hedges, L. V., \& Olkin, I. (1985). Statistical methods for meta-analysis. Academic Press.

Juslin, P., Olsson, N., \& Winman, A. (1996). Calibration and diagnosticity of confidence in eyewitness identification: Comments on what can be inferred 
from the low confidence-accuracy correlation. Journal of Experimental Psychology: Learning Memory and Cognition, 22(5), 1304-1316. https://doi.org/10.1037/0278-7393.22.5.1304

Loftus, E. F., \& Greenspan, R. L. (2017). If I'm Certain, Is It True? Accuracy and Confidence in Eyewitness Memory. Psychological Science in the Public Interest, 18(1), 1-2. https://doi.org/10.1177/1529100617699241

Penrod, S., \& Cutler, B. (1995). Witness Confidence and Witness Accuracy: Assessing Their Forensic Relation. Psychology, Public Policy, and Law, 1(4), 817-845. https://doi.org/10.1037/1076-8971.1.4.817

Pescetelli, N., \& Yeung, N. (2021). The role of decision confidence in advice-taking and trust formation. Journal of Experimental Psychology: General, 150(3), 507526. https://doi.org/10.1037/xge0000960

Rahnev, D., Desender, K., Lee, A. L. F., Adler, W. T., Aguilar-Lleyda, D., Akdoğan, B., Arbuzova, P., Atlas, L. Y., Balcı, F., Bang, J. W., Bègue, I., Birney, D. P., Brady, T. F., Calder-Travis, J., Chetverikov, A., Clark, T. K., Davranche, K., Denison, R. N., Dildine, T. C., ... Zylberberg, A. (2020). The Confidence Database. Nature Human Behaviour, 4(3), 317-325. https://doi.org/10.1038/s41562-019-0813-1

Shekhar, M., \& Rahnev, D. (2021a). Sources of Metacognitive Inefficiency. Trends in Cognitive Sciences, 25(1), 12-23. https://doi.org/10.1016/j.tics.2020.10.007

Shekhar, M., \& Rahnev, D. (2021b). The nature of metacognitive inefficiency in perceptual decision making. Psychological Review, 128(1), 45-70. https://doi.org/10.1037/rev0000249

Sporer, S. L., Penrod, S., Read, D., \& Cutler, B. (1995). Choosing, Confidence, and 
Accuracy: A Meta-Analysis of the Confidence-Accuracy Relation in Eyewitness Identification Studies. Psychological Bulletin, 118(3), 315-327. https://doi.org/10.1037/0033-2909.118.3.315

Tenney, E. R., Spellman, B. A., \& MacCoun, R. J. (2008). The benefits of knowing what you know (and what you don't): How calibration affects credibility. Journal of Experimental Social Psychology, 44(5), 1368-1375. https://doi.org/10.1016/j.jesp.2008.04.006

Viechtbauer, W. (2010). Conducting Meta-Analyses in R with the metafor Package. Journal of Statistical Software, 36(1), 1-48. https://doi.org/10.18637/JSS.V036.I03

Wixted, J. T., \& Wells, G. L. (2017). The Relationship Between Eyewitness Confidence and Identification Accuracy: A New Synthesis. Psychological Science in the Public Interest, 18(1), 10-65. https://doi.org/10.1177/1529100616686966

Yang, H., \& Thompson, C. (2010). Nurses' risk assessment judgements: A confidence calibration study. Journal of Advanced Nursing, 66(12), 2751-2760. https://doi.org/10.1111/j.1365-2648.2010.05437.x

Zell, E., \& Krizan, Z. (2014). Do People Have Insight Into Their Abilities? A Metasynthesis. Perspectives on Psychological Science, 9(2), 111-125. https://doi.org/10.1177/1745691613518075 